\title{
Distribuição espacial das guildas tróficas e estruturação da comunidade de Ephemeroptera (Insecta) em córregos do Cerrado de Mato Grosso, Brasil
}

\author{
Yulie Shimano ${ }^{1}$, Frederico F. Salles ${ }^{2}$, Luiz R. R. Faria ${ }^{2}$, Helena S. R. Cabette ${ }^{1} \&$ Denis S. Nogueira ${ }^{3}$ \\ 1. Departamento de Ciências Biológicas, Universidade do Estado de Mato Grosso, Nova Xavantina, MT, Brasil. (yulie.bio@gmail.com, hcabette@uol.com.br) \\ 2. Departamento de Ciências Agrárias e Biológicas, Universidade Federal de Espírito Santo, Centro Universitário Norte do Espírito Santo, São Mateus, ES, Brasil. (ffsalles@gmail.com, \\ nunofariajr@gmail.com) \\ 3. Programa de Pós-Graduação em Ecologia e Evolução, Universidade Federal do Goiás, Goiânia, GO, Brasil. (dnogueira.bio@gmail.com)
}

\begin{abstract}
Spatial distribution of trophic guilds and community structure of Ephemeroptera (Insecta) in streams of the Cerrado region in Mato Grosso, Brazil. We analyzed the structure of the functional feeding groups of Ephemeroptera in five different types of substrates (litter in riffles, backwater litter, wood, stones and roots) from first to fifth orders at the Pindaíba River basin, state of Mato Grosso, Brazil. We also studied the specificity and frequency of the organism, and if the functional approach reflects changes within the community through ordination analysis. Our results assemble information about the functional classification of mayfly nymphs and show that scrapers were the most abundant, and gathering - collectors are the more diverse. Third order streams were the most richest and most abundant while functional feeding group distribution corroborated the predictions of The River Continuum Concept. Besides, communities were much more taxonomically structured than functionally structured. This fact underlies the importance of the biology of organisms, and not only the functional feeding group, for substrate selection. The preference of some genera for specific substrates confirms this value.
\end{abstract}

KEYWORDS. Taxonomic composition, aquatic insect, mesodistribution, indicator species.

RESUMO. Analisamos a estrutura dos grupos funcionais alimentares de Ephemeroptera em cinco tipos de substratos (folhiço em corredeira, folhiço em remanso, madeira, pedras e raízes) de trechos de primeira a quinta ordens da bacia do rio Pindaíba, estado do Mato Grosso, Brasil. Verificamos, ainda, a preferência de táxons por substratos específicos através da especificidade e frequência dos organismos, e se a abordagem funcional reflete as variações dentro da comunidade. Nossos resultados agregam informações a respeito da classificação funcional dos efemerópteros e mostram que os raspadores foram os mais abundantes e os coletores-apanhadores comportam uma maior riqueza de táxons. Os córregos de terceira ordem foram os mais ricos e abundantes enquanto que as distribuições dos grupos funcionais alimentares corroboram as predições do Conceito do Continuum fluvial. Além disso, as comunidades estudadas foram muito mais estruturadas taxonomicamente do que funcionalmente. Esse fato atesta a importância da biologia dos organismos para a seleção dos substratos. A preferência de alguns gêneros por substrato específico corrobora esta importância.

PALAVRAS-CHAVE. Composição taxonômica, insetos aquáticos, mesodistribuição, espécies indicadoras.

O conceito de guilda alimentar foi proposto originalmente por Rоoт (1967), definido como um grupo de espécies, indiferente da afiliação taxonômica, que exploram a mesma classe de recursos alimentares de uma maneira similar. Quando insetos aquáticos são divididos em guildas tróficas, essas categorias são conhecidas como grupos funcionais alimentares (Functional Feeding Groups - FFG), e são baseadas não só no tipo de recurso consumido, mas também nos mecanismos morfológicos e comportamentais de aquisição desses recursos (Cummins, 1973; Cummins \& Klug, 1979; Cummins et al., 2008). De acordo com a classificação proposta por Cummins (1973), os insetos aquáticos podem ser classificados como coletoresapanhadores, coletores-filtradores, fragmentadores, predadores ou raspadores.

As proporções dos grupos funcionais alimentares em um ecossistema podem variar de acordo com o tamanho do rio e o tipo de substrato disponível. Por exemplo, o conceito do continuum fluvial, proposto por VANNOTE et al. (1980), prediz que a distribuição das guildas tróficas estaria diretamente ligada à disponibilidade de alimento e energia ao longo do rio e intimamente ligada à influência da vegetação ripária sobre os cursos d'água. Nas cabeceiras, devido à grande entrada de matéria orgânica provinda da densa vegetação, haveria maior biomassa de fragmentadores e de coletores, que consumiriam matéria particulada pelos fragmentadores. Em trechos de tamanhos intermediários haveria maior biomassa de raspadores devido à produção autotrófica, que começa a partir da entrada de luz no sistema, e de coletores, que ainda permaneceriam com a mesma proporção que nas cabeceiras. Finalmente, em trechos mais largos haveria um aumento da biomassa de coletores, uma vez que a matéria orgânica finamente particulada vinda de trechos a montante seria o principal componente alimentar. Os predadores seriam os menos representativos, mas manteriam a mesma proporção de biomassa ao longo do contínuo devido à manutenção da disponibilidade de presas (VANNOTE et al., 1980).

Da mesma forma, em se tratando de associações dos grupos funcionais alimentares com tipos diferentes de substratos, espera-se que organismos coletores-filtradores se estabeleçam em substratos associados a fluxos de água, onde o movimento das partículas orgânicas lhes proporcionaria coletar alimento na coluna d'água. Os coletores-apanhadores, pelo contrário, provavelmente estariam associados a substratos com baixo fluxo de água, que permite o depósito das partículas de alimento. Já os fragmentadores, estariam associados a substratos orgânicos maiores, uma vez que se alimentam diretamente deles, enquanto que os raspadores deveriam se estabelecer 
em pedras e madeiras onde poderiam se alimentar do perifíton associado (Cummins et al., 2005). A abundância, riqueza e composição de insetos aquáticos também são parâmetros fortemente influenciados pelos tipos de substratos disponíveis (Minshall, 1984; HANNAFORD et al., 1997; Hilsenhoff, 2001; Schowalter, 2006).

Os grupos funcionais alimentares, dessa forma, têm sido muito utilizados em estudos ecológicos de insetos aquáticos devido à amplitude de informações que representam (FrancischetTI et al., 2004; МоtTA \& UiedA, 2004; Kikuchi \& UiedA, 2005). Avaliar a comunidade de macroinvertebrados através de sua estrutura trófica permite algumas vantagens: (1) gera a percepção de qual recurso alimentar é o que prevalece no sistema; (2) possibilita observar como diferentes grupos de organismos respondem às variáveis ambientais; e, (3) uma vez que a identificação no nível específico requer tempo, treino e prática, a utilização de grupos funcionais alimentares diminui a magnitude de classes, se apresentando como método alternativo para estudos de comunidade (Allan \& Castillo, 2007; Cummins et al., 2008). A abordagem funcional provou ser um sistema de classificação útil e durável para a caracterização da condição do ecossistema, sendo mais apropriada do que a abordagem taxonômica (VANNOTE et al., 1980; Cummins et al., 2005). Por outro lado, de acordo com HilsenHOFF (2001), as desvantagens de tal abordagem são que o tipo de alimentação pode variar de acordo com a disponibilidade de alimento e que os hábitos alimentares podem variar ao longo do crescimento dos organismos.

Efemerópteros mostram-se úteis e importantes para estudos tróficos uma vez que constituem um dos principais componentes da fauna de macroinvertebrados bentônicos (Hilsenhoff, 2001; Domínguez et al., 2006) e possuem integrantes em todas as categorias funcionais alimentares. Dessa forma, nossos objetivos são: (i) analisar a composição de espécies e a estrutura dos grupos funcionais alimentares em diferentes ordens de córregos e rios; (ii) analisar a composição de espécies e a estrutura dos grupos funcionais alimentares em diferentes tipos de substratos, verificando associações por substratos específicos; e (iii) avaliar se a abordagem funcional de fato reflete as variações na estrutura das comunidades. Esperamos que tanto a composição quanto a distribuição de grupos funcionais alimentares variem de acordo com o continuum fluvial e de acordo com os tipos de substratos.

\section{MATERIAL E MÉTODOS}

Nosso estudo foi realizado na bacia do rio Pindaíba, leste do estado de Mato Grosso, Brasil

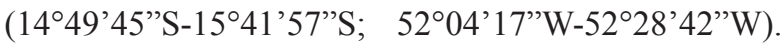
Essa bacia é tributária do Rio das Mortes, e se localiza nos municípios de Araguaiana, Barra do Garças, Cocalinho e Nova Xavantina.

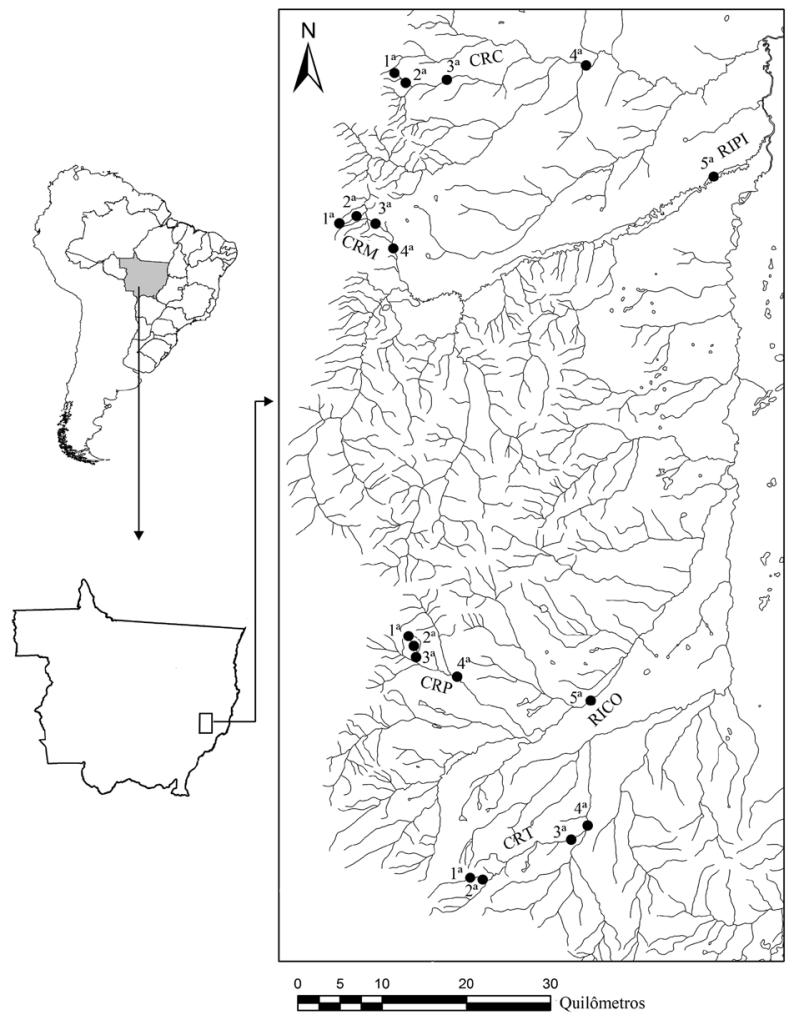

Fig. 1. Pontos de coleta de imaturos de Ephemeroptera na Bacia do Rio Pindaíba, Mato Grosso, e suas respectivas ordens (CRC, Córrego Cachoeirinha; CRM, Córrego da Mata; CRP, Córrego Papagaio; CRT, Córrego Taquaral; RICO, Rio Corrente; RIPI, Rio Pindaíba).

De acordo com a classificação de Köppen, o clima é do tipo $C w a$, com duas estações climáticas bem definidas, um período seco e outro período chuvoso. As médias anuais de precipitação variam de 1200 a 1600 $\mathrm{mm}$, e a temperatura média varia em torno de 20 a $25^{\circ} \mathrm{C}$, sendo que os meses mais quentes são setembro e outubro (BrasiL, 1981).

As ninfas de Ephemeroptera foram coletadas em córregos e rios de primeira a quinta ordens ao longo da bacia do rio Pindaíba (Fig. 1). Em quatro córregos (Cachoeirinha, da Mata, Papagaio e Taquaral) foram amostrados trechos de primeira a quarta ordem, enquanto que as amostras de quinta ordem foram realizadas nos rios Pindaíba e Corrente, correspondendo ao todo, a 18 pontos amostrados. As coletas foram realizadas em três períodos do ano: início (outubro e novembro) e meio (janeiro) da estação chuvosa, e na estação seca (julho e agosto) de 2005, resultando em 12 amostras para cada córrego de até quarta ordem e seis amostras nos rios de quinta ordem. Para determinação das ordens dos rios seguiu-se a classificação proposta por STRAHLER (1957).

Foram selecionados cinco tipos de substratos a serem amostrados em 100 metros lineares de cada córrego e rio: folhiços depositado em áreas de corredeira, folhiços depositado em áreas de remanso, madeira, pedra (matacão) e raiz, sendo estes definidos como manchas homogêneas de mesohábitats visualmente distinguíveis quando se observa da margem ou do leito do riacho, como 
proposto por PARDo \& ARmitage (1997). Madeira, pedra e raiz foram amostradas tanto em áreas de corredeira quanto em áreas de remanso. Os substratos orgânicos foram obtidos com um coador ("rapiché" de $18 \mathrm{~cm}$ de diâmetro e malha de $250 \mathrm{~mm}$ ), de forma que três coadores por mancha de mesohábitat foram considerados uma amostra e cada uma foi replicada cinco vezes em cada local (Assis et al., 2004 utilizou quatro subamostras). Como a distribuição dos substratos pedra e madeira foi muito irregular nos trechos amostrados e não permitiu o uso do coador, optou-se por padronizar a amostragem de organismos nestes substratos por tempo. Assim, testou-se o tempo necessário para percorrer e amostrar manchas existentes desses substratos nos 100 metros lineares e estabeleceu-se esse tempo como padrão nas coletas subsequentes para manter tamanhos amostrais similares. Sendo assim, madeira e pedra (tamanho dos matacões baseados em Melo \& Frohlich, 2001), foram amostradas manualmente durante 15 minutos com auxílio de pinças e escovas. Não se utilizou o método tradicional de coleta com surber, por não permitir isolar cada substrato nas amostragens.

Os imaturos foram identificados em nível de gênero, com auxílio da chave de identificação (DomínGUEZ et al., 2006). Em seguida, os gêneros foram classificados de acordo com sua categoria funcional (Cummins, 1973; 1974), a partir de informações contidas em artigos (Tab. I). Informações baseadas em outras classificações funcionais (e.g. MCShaffrey \& MCCAFFerTy, 1988; PALMER et al., 1993), que apresentam categorias mais específicas, foram simplificadas nos grupos funcionais alimentares: coletor-apanhador, coletorfiltrador, fragmentador, raspador e predador propostos por Cummins (1973; 1974). Quando as informações divergiam, foram consideradas as categorias citadas por mais de um autor. No caso de não ser encontrado o comportamento alimentar na literatura, a categoria alimentar foi inferida com base nas informações morfológicas e comportamentais observadas em campo. Quando o grupo funcional citado pela bibliografia divergia do esperado (baseado em observações de campo), foi realizada uma análise do conteúdo estomacal, onde a parte superior do trato digestivo era retirada, aberta e o conteúdo colocado em lâmina com glicerina, sobreposta por uma lamínula e observada em microscópio óptico (aumento de até 1000×).

Todos os espécimes utilizados nas análises foram depositados na Coleção Zoobotânica "James Alexander Ratter", Universidade do Estado de Mato Grosso, UNEMAT, campus de Nova Xavantina (CZNX, curadora H.S.R. Cabette).

Foram utilizadas análises de variância (ANOVA) para comparar a abundância de indivíduos de acordo com as ordens dos córregos e rios e a abundância dos grupos funcionais alimentares dentro dos diferentes tipos de substratos. Quando significativos ( $p<0,05$ ), contrastamos a significância entre as ordens de magnitude por meio do
Teste de Tukey, como análise a posteriori para indicar a diferença entre os pares de ordens (ZAR, 1999). As estações do ano foram utilizadas como réplica e os dados de abundância foram logaritmizados nas análises entre grupos funcionais.

A riqueza de gêneros entre as ordens dos ambientes foi comparada com base na técnica de rarefação de Mao Tau ("baseada em momentos") (Gotelli \& Colwell, 2001). Devido aos diferentes métodos empregados na coleta dos Ephemeroptera, foi necessário usar um método que não se fundamente na abundância das amostras. Esse método permite a comparação direta de riqueza entre amostras (Colwell et al., 2004). Como as ordens dos córregos e rios diferiram quanto ao número de córregos amostrados, foram consideradas apenas 30 amostras por ordem (cinco tipos de substratos $\times$ quatro córregos $\times$ três estações $=30$ amostras). A rarefação foi calculada no programa EstimateS 7.5.0 (Colwell, 2005).

A composição de gêneros e grupos funcionais alimentares nas diferentes ordens de córregos e rios foi verificada através de ordenação usando análises de correspondência destendenciada com remoção do efeito do arco (Detrented Correspondence Analysis - DCA) (Hill \& GAUCH, 1980) a partir do log da abundância das comunidades. $\mathrm{O}$ mesmo foi realizado para verificar a composição nos diferentes tipos de substratos. Em seguida, para testar a significância dos agrupamentos estabelecidos pelas DCAs, foram realizadas análises de variância (ANOVA) com os primeiros eixos estabelecidos.

Para todas as análises realizadas com os grupos funcionais alimentares, o grupo predador foi retirado, já que apenas um exemplar foi coletado.

Para verificar se os gêneros apresentaram associação por algum tipo de substrato específico, foi utilizada a análise de espécies indicadoras (Indicator Value Method - IndVal) (Dufrêne \& Legendre, 1997), com nível de significância de 5\%. De acordo com os autores, esse índice mede o grau de especificidade (relação da espécie com uma variável específica) e o grau de fidelidade (toda vez que a variável ocorre, a espécie está presente) em relação a uma categoria ambiental.

\section{RESULTADOS}

Foram coletadas 2173 ninfas de Ephemeroptera, distribuídas em sete famílias e 35 gêneros. Baetidae e Leptophlebiidae registraram o maior número de gêneros amostrados, ambas com 12 gêneros, e Leptophlebiidae foi a mais abundante, contribuindo com mais de $63 \%$ da abundância total de indivíduos. Farrodes Peters, 1971, Miroculis Edmunds, 1963 e Traverhyphes Molineri, 2001 foram os gêneros mais abundantes $(559,415$ e 301 indivíduos, respectivamente). Amanahyphes Salles \& Molineri, 2006 e Harpagobaetis Mol, 1986 foram representados por apenas um indivíduo cada. 
Tab. I. Famílias e gêneros de Ephemeroptera coletados na Bacia do Rio Pindaíba, Mato Grosso, em trechos de primeira a quinta ordem, e respectivos grupos funcionais alimentares (GFA) de acordo com a bibliografia citada (*inferência baseada na categoria dos organismos da mesma linhagem; **baseado no conteúdo estomacal; CA, coletor-apanhador; CF, coletor-filtrador; F, fragmentador; P, predador; R, raspador).

\begin{tabular}{|c|c|c|}
\hline Família/Gênero & GFA & Fonte \\
\hline \multicolumn{3}{|l|}{ Baetidae } \\
\hline Americabaetis & $\mathrm{CA}$ & Cummins et al., 2005; Merrit et al., 2008 \\
\hline Apobaetis & $\mathrm{CA}$ & Cummins et al., 2005 \\
\hline Aturbina & $\mathrm{CA}$ & Cummins et al., 2005 \\
\hline Baetodes & $\mathrm{R}$ & BAPTISTA et al., 2006; MERRIT et al., 2008 \\
\hline Camelobaetidius & $\mathrm{R}$ & BAPTISTA et al., 2006 \\
\hline Cloeodes & $\mathrm{R}$ & BAPTISTA et al., 2006 \\
\hline Cryptonympha & $\mathrm{CA}$ & Cummins et al., 2005 \\
\hline Guajirolus & $\mathrm{CA}$ & Cummins et al., 2005 \\
\hline Harpagobaetis & $\mathrm{P}$ & MoL, 1986 \\
\hline Paracloeodes & $\mathrm{CA}$ & Cummins et al., 2005 \\
\hline Waltzoyphius & $\mathrm{CA}$ & Cummins et al., 2005 \\
\hline Zelusia & $\mathrm{CA}$ & Cummins et al., 2005 \\
\hline \multicolumn{3}{|l|}{ Caenidae } \\
\hline Brasilocaenis & $\mathrm{R}$ & * \\
\hline Caenis & $\mathrm{R}$ & Francischetti et al., 2001; MerRit et al., 2008 \\
\hline \multicolumn{3}{|l|}{ Euthyplociidae } \\
\hline Campylocia & $\mathrm{CA}$ & Cummins et al., 2005 \\
\hline \multicolumn{3}{|l|}{ Leptohyphidae } \\
\hline Amanahyphes & $\mathrm{CA}$ & Cummins et al., 2005 \\
\hline Leptohyphes & $\mathrm{CA}$ & Molineri, 2003; Cummins et al., 2005; Merrit et al., 2008 \\
\hline Traverhyphes & $\mathrm{CA}$ & Cummins et al., 2005 \\
\hline Tricorythodes & $\mathrm{CA}$ & Cummins et al., 2005; Merrit et al., 2008 \\
\hline Tricorythopsis & $\mathrm{CA}$ & Cummins et al., 2005 \\
\hline \multicolumn{3}{|l|}{ Leptophebiidae } \\
\hline Askola & $\mathrm{R}$ & Polegatto \& Froehlich, 2003 \\
\hline Farrodes & $\mathrm{R}$ & Polegatto \& Froehlich, 2003 \\
\hline Fittkaulus & $\mathrm{F}$ & $*$ \\
\hline Hagenulopsis & $\mathrm{R}$ & Polegatto \& Froehlich, 2003; Baptista et al., 2006 \\
\hline Hydrosmilodon & $\mathrm{CF}$ & Polegatto \& Froehlich, 2003 \\
\hline Miroculis & $\mathrm{R}$ & Polegatto \& Froenlich, 2003 \\
\hline Paramaka & $\mathrm{CF}$ & $*$ \\
\hline Simothraulopsis & $\mathrm{R}$ & $*$ \\
\hline Terpides & $\mathrm{F}$ & BELLo \& CABRERA, 2001 \\
\hline Thraulodes & $\mathrm{R}$ & Polegatto \& Froehlich, 2003; Baptista et al., 2006; Merrit et al., 2008 \\
\hline Traverella & $\mathrm{CF}$ & Edmunds et al., 1976; Polegatto \& Froehlichi, 2003; Merrit et al., 2008 \\
\hline Ulmeritoides & $\mathrm{F}$ & $* *$ \\
\hline \multicolumn{3}{|l|}{ Oligoneuriidae } \\
\hline Lachlania & $\mathrm{CF}$ & Cummins et al., 2005; Merrit et al., 2008 \\
\hline Oligoneuria & $\mathrm{CF}$ & Cummins et al., 2005 \\
\hline \multicolumn{3}{|l|}{ Polymirtacyidae } \\
\hline Campsurus & $\mathrm{CF}$ & EDMUNDS et al., 1976; HiLSENHOFF, 2001 \\
\hline
\end{tabular}

O grupo funcional mais representativo foi dos raspadores, contribuindo com mais de $56 \%$ dos indivíduos amostrados $(\mathrm{n}=1234)$ em 11 gêneros. Os coletores-apanhadores somaram $32,4 \%$ dos indivíduos $(n=705)$ distribuídos em 14 gêneros, enquanto que coletores-filtradores (seis gêneros), fragmentadores (três gêneros) e predadores (um gênero e um indivíduo) somaram pouco mais de $10 \%$ da abundância total. Houve diferenças significativas entre as proporções de grupos funcionais $\left(\mathrm{F}_{(3,56)}=34,498 \mathrm{e} \mathrm{p}<0,001\right)$, sendo que os raspadores foram mais abundantes que coletoresfiltradores, fragmentadores, e coletores-apanhadores mais abundantes que coletores-filtradores e predadores (Tukey, $\mathrm{p}<0,05$ ).

$\mathrm{O}$ grupo funcional alimentar que mais contribuiu para a riqueza de gêneros foi o dos coletores-apanhadores (14 gêneros), seguido dos raspadores (11 gêneros), os coletores-filtradores (seis gêneros), fragmentadores (três gêneros) e por fim, o grupo de predadores, que contribuiu com apenas um gênero.

Quanto à distribuição ao longo da bacia do rio Pindaíba (Fig. 2), a abundância foi diferente entre as ordens analisadas $\left(\mathrm{F}_{(4,20)}=4,771\right.$ e $\left.\mathrm{p}=0,007\right)$. A terceira ordem apresentou maior abundância, obtendo em média 

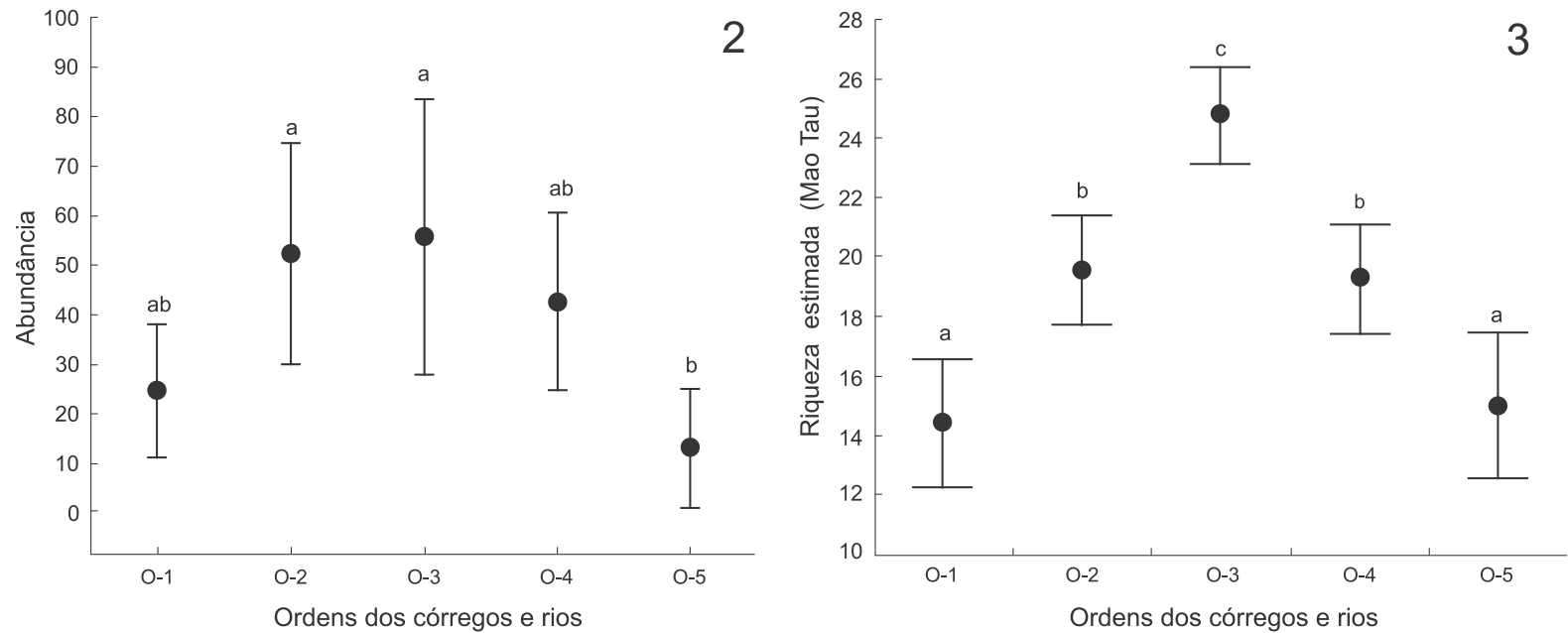

Figs 2, 3. Análise de variância com a abundância (2) e riqueza observada (Mao Tau) de gêneros de Ephemeroptera (3) em trechos de córregos de primeira à quinta ordem da Bacia do Rio Pindaíba, Mato Grosso, 2005 (Barras representam desvio padrão e intervalo de confiança de 95\%).

56 indivíduos por amostra, seguida da segunda ordem (53 indivíduos por amostra), quarta $(\mathrm{n}=43)$, primeira $(\mathrm{n}=41)$ e a quinta $(\mathrm{n}=13)$. Porém, a diferença se deu apenas entre a segunda e terceira ordens quando comparadas individualmente com a quinta ordem (Tukey, $\mathrm{p}<0,05$ ).

As riquezas de gêneros também diferiram entre si (Fig. 3). A terceira ordem foi a mais rica (riqueza, 24,0 ; intervalo de confiança $\pm 1,6$ ), diferindo da segunda $(19,5 \pm 1,8)$ e quarta ordens $(19,2 \pm 2,4)$, que por sua vez, diferiram da quinta $(15,0 \pm 2,4)$ e primeira ordens $(14,4 \pm 2,1)$.

Os coletores-apanhadores apresentaram variação em sua distribuição ao longo do gradiente de largura, sendo mais representativos na quinta ordem, enquanto que os coletores-filtradores tiveram sua proporção aumentando de acordo com o aumento da ordem dos rios (Figs 4, 5, respectivamente). Os fragmentadores obtiveram sua maior proporção em trechos de cabeceira, diminuindo em relação à foz, obtendo pequeno aumento na quarta ordem (Fig. 6). Por fim, os raspadores tiveram maior abundância relativa em trechos de segunda ordem (Fig. 7).

Ao relacionar os grupos funcionais alimentares e as ordens dos rios através da DCA, apenas $29,06 \%$ da variação foi explicada nos dois primeiros eixos (eixo $1=23,4 \%$ e eixo $2=5,6 \%$ ) e não foi observado nenhum

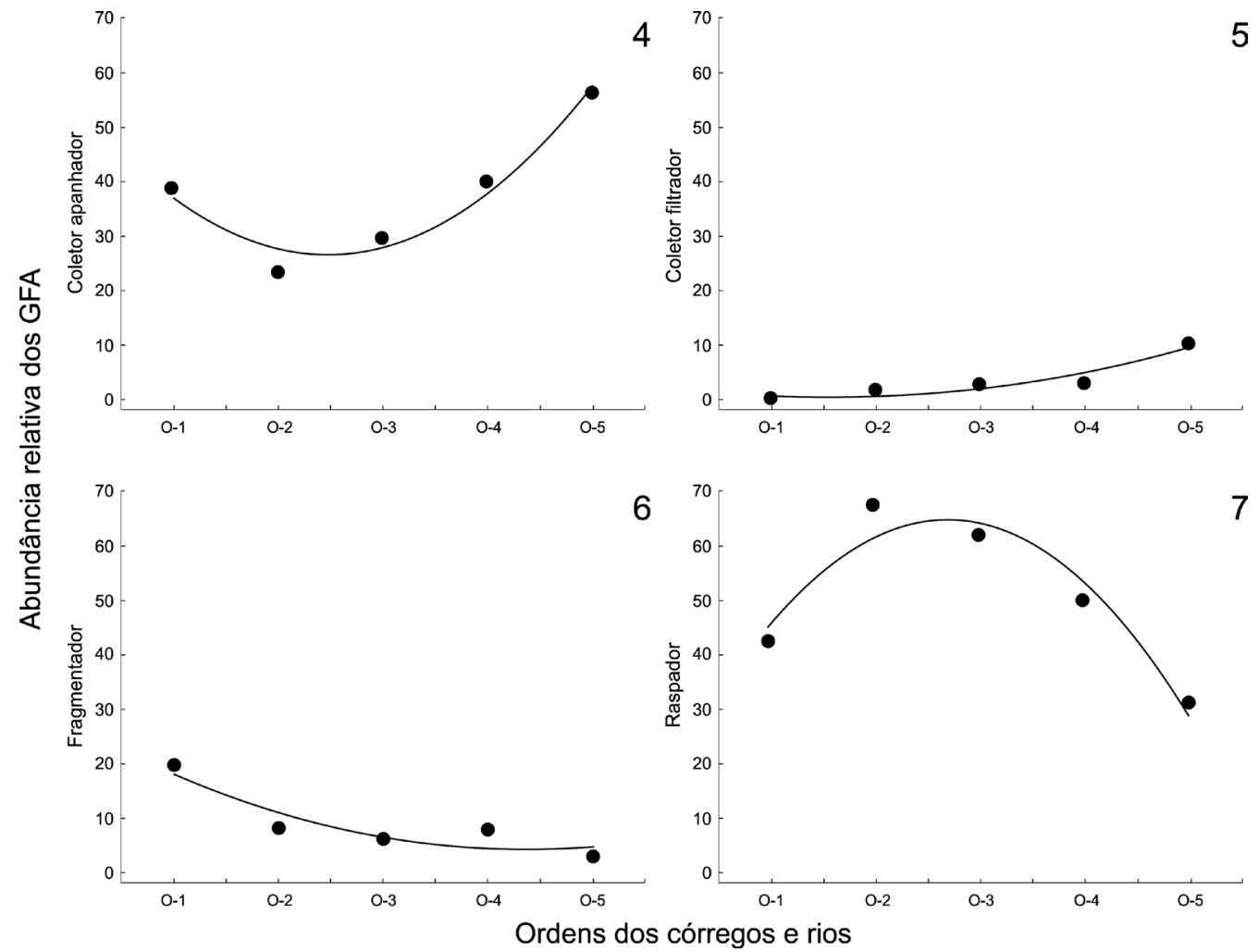

Figs 4-7. Abundância relativa dos grupos funcionais alimentares de Ephemeroptera em trechos de córregos de primeira à quinta ordem da Bacia do Rio Pindaíba, Mato Grosso, 2005: 4, coletores-apanhadores; 5, coletores-filtradores; 6, fragmentadores; 7, raspadores. 
padrão de agrupamento entre os grupos funcionais alimentares e as ordens, o que foi confirmado pela $\operatorname{ANOVA}\left(\mathrm{F}_{(3,30)}=2,073, \mathrm{p}=0,125\right)$.

Ao ordenar a composição de gêneros a partir das ordens nos córregos, 55,14\% da variabilidade total da composição foi explicada com os dois primeiros eixos (eixo $1=32,95 \%$; eixo $2=22,19 \%$ ). Não foram observados agrupamentos entre as ordens, porém, a ANOVA mostrou diferenças significativas na composição de gêneros $\left(F_{(4,19)}=4,175, p=0,014\right)$.

Ao analisar a distribuição funcional dentro dos substratos amostrados, observou-se que todos os substratos apresentaram diferenças com relação à abundância $(p<0,05)$ (Figs 8-12). Folhiço em corredeira e raiz apresentaram as médias de abundância de coletores-filtradores e fragmentadores menores do que a dos coletores-apanhadores e raspadores (Figs 8, 12); folhiço em remanso e pedra apresentaram os
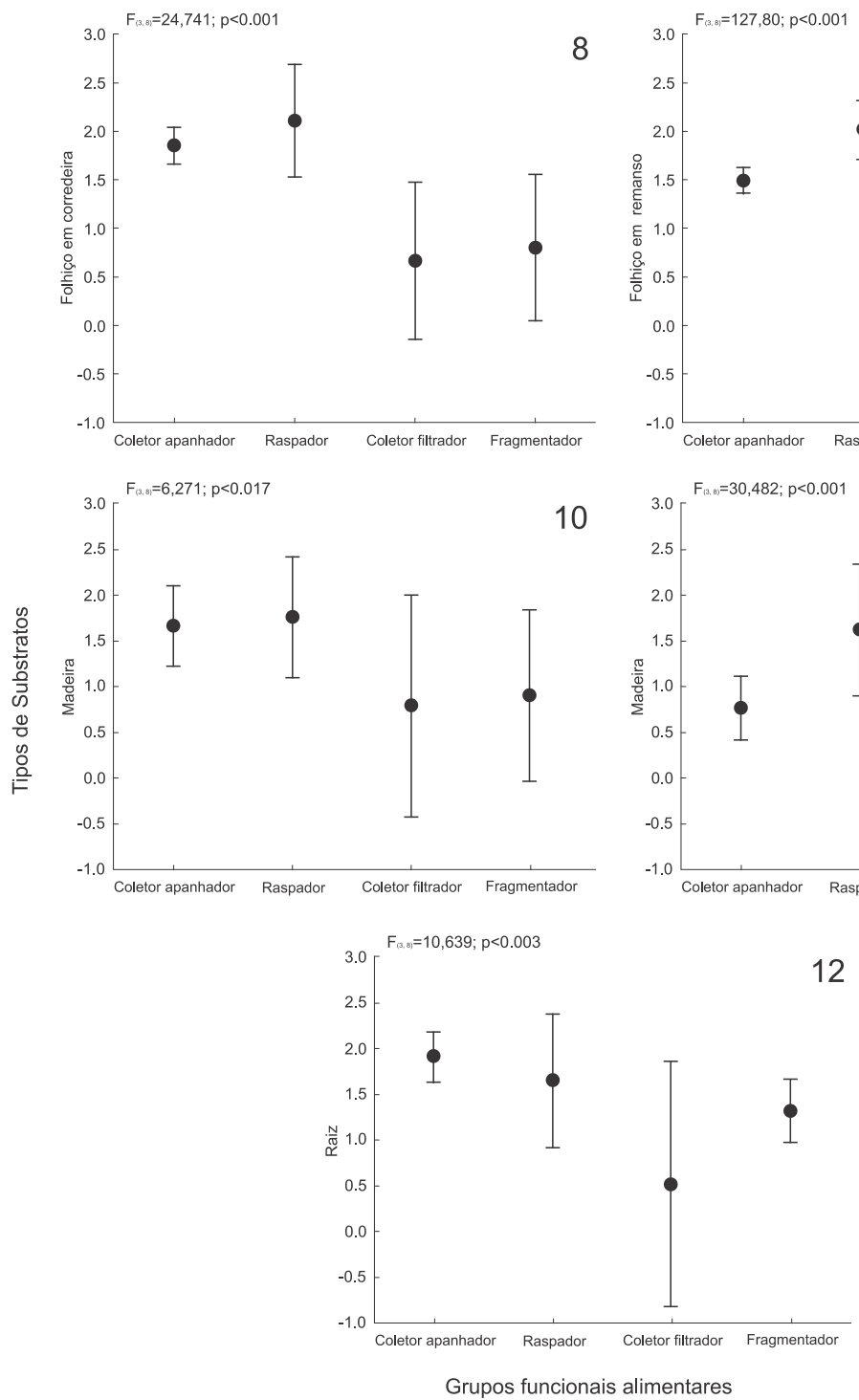

Figs 8-12. Análise de variância do log da abundância dos grupos funcionais alimentares de Ephemeroptera de acordo com os diferentes tipos de substrato da Bacia do Rio Pindaíba, Mato Grosso, 2005: 8, folhiço em remanso; 9, folhiço em corredeira; 10, madeira; 11, pedra; 12, raiz (barras desvio padrão de $95 \%$ ).
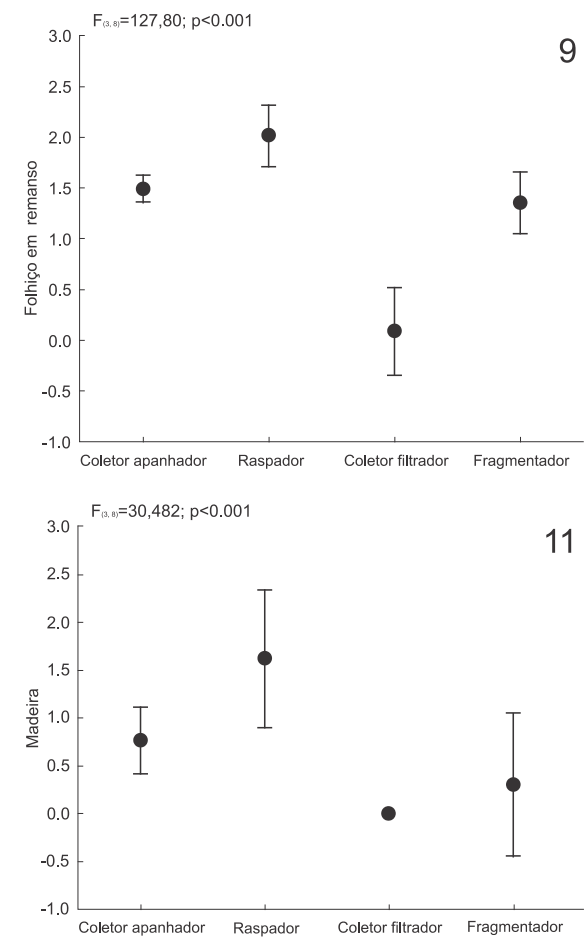

fragmentadores com abundância média menor do que a de coletores-apanhadores (Figs 9, 11) enquanto que madeira apresentou a abundância de filtradores menor do que a de raspadores (Tukey, p<0,05; Fig. 10).

A distribuição da maioria dos grupos funcionais nos substratos apresentou o mesmo perfil, com os raspadores sempre como os mais abundantes, seguidos de coletores-apanhadores, fragmentadores e coletoresfiltradores (Figs 8-11).

Ao ordenar os grupos funcionais alimentares de acordo com tipo de substrato, os dois primeiros eixos da DCA explicaram 32,90\% da variação total (eixo $1=20,17 \%$ e eixo $2=12,73 \%$ ). Não foi observado agrupamento entre os gêneros. A análise de variância (ANOVA) com o primeiro eixo da DCA também não detectou diferenças nos grupos funcionais alimentares $\left(\mathrm{F}_{(3,30)}=1,089, \mathrm{p}=0,369\right)$.

No entanto, ao ordenar os substratos a partir da

2 
composição de gêneros associada a cada um deles, a ordenação foi mais significativa $(55,14 \%$ da variabilidade total da composição explicada pelos dois primeiros eixos; eixo $1=32,95 \%$; eixo $2=22,19 \%$ ). Percebe-se um claro agrupamento entre as amostras de folhiço em remanso e folhiço em corredeira (Fig. 13). As amostras realizadas nos substratos madeira, pedra e raiz não apresentaram agrupamentos visíveis. Os agrupamentos foram confirmados pela ANOVA $\left(\mathrm{F}_{(4,19)}=3,285, \mathrm{p}=0,033\right)$.

A análise de espécies indicadoras mostrou a associação de alguns gêneros com substratos específicos. Thraulodes Ulmer, 1920 apresentou valor alto de indicação por pedra, Fittkaulus Savage \& Peters, 1978 por raiz e Baetodes Needhan \& Muphy, 1924 apresentou indicação por dois tipos de substratos, folhiço em corredeira e pedra (Tab. II).

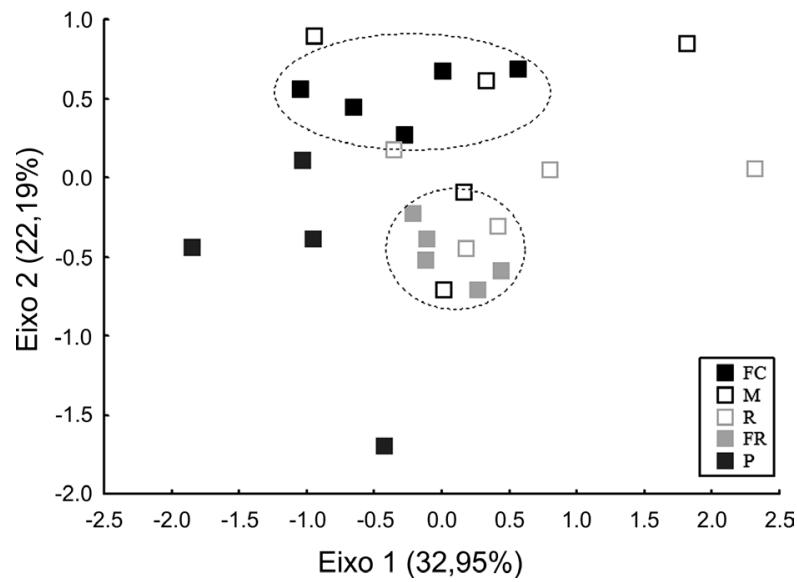

Fig. 13. Ordenação da análise de correspondência com remoção do efeito do arco (DCA) das amostras de substratos baseado na composição de gêneros em córregos da Bacia do Rio Pindaíba, Mato Grosso, 2005 (FC, folhiço/corredeira; FR, folhiço/remanso; M, madeira; P, pedra; $\mathrm{R}$, raiz)

Tab. II. Gêneros, categoria funcional alimentar (FFG), valor de indicação, teste (p) e indicação de substrato específico da Análise de Espécies Indicadoras, realizada para os Ephemeroptera imaturos da Bacia do Rio Pindaíba, Mato Grosso, 2005.

\begin{tabular}{|c|c|c|c|c|}
\hline Gênero & FFG & $\begin{array}{l}\text { Valor de } \\
\text { indicação }\end{array}$ & $\mathrm{p}^{*}$ & Indicação \\
\hline Thraulodes & Raspador & 83,6 & 0,008 & Pedra \\
\hline Fittkaulus & Fragmentador & 86,6 & 0,001 & Raiz \\
\hline Baetodes & Raspador & 71,6 & 0,050 & $\begin{array}{c}\text { Folhiço de } \\
\text { Corredeira }+ \\
\text { Pedra }\end{array}$ \\
\hline
\end{tabular}

\section{DISCUSSÃO}

Nossas hipóteses de variação da composição e distribuição dos grupos funcionais de acordo com o predito pelo conceito de continuum fluvial (VANNOTE et al., 1980), foram parcial ou totalmente corroboradas. Verificamos que, se não para todos, pelo menos para a maioria dos grupos funcionais, a distribuição segue o predito pelo conceito de continuum fluvial. Os raspadores corresponderam a mais da metade dos organismos e foram os mais abundantes em todos os tipos de substratos amostrados. Esse resultado pode ser atribuído à grande abundância de dois gêneros raspadores, Farrodes e Miroculis, os quais corresponderam à cerca de $40 \%$ da abundância total de indivíduos. Ambos os gêneros possuem ampla distribuição e são encontrados em substratos orgânicos e inorgânicos de diversos tipos (folhas em poções ou em corredeiras, raízes, pedras), além de aparentemente possuírem ampla tolerância e compor grande parte da fauna de efemerópteros em riachos de várias regiões no Brasil (SAVAGE, 1987; Domínguez et al., 2006). A própria família Leptophlebiidae como um todo é considerada um elemento dominante na fauna de efemerópteros em córregos de pequeno e médio porte da Região Neotropical (SAVAGE, 1987), e sua alta abundância tem sido amplamente registrada (SALLES et al., 2004; Siegloch et al., 2008; Shimano et al., 2010; SALLes, 2011).

Além disso, em uma classificação mais específica, organismos da família Leptophlebiidae podem ser enquadrados dentro de raspadores como escovadores (F. F. Salles, com. pess.). Esses organismos caracterizamse por alimentarem-se de matéria orgânica particulada fina (MOPF) e algas/perifíton ligeiramente fixadas nos substratos, enquanto que os raspadores (stricto sensu) possuem adaptações estruturais para alimentarem-se de MOPF e algas/perifíton firmemente aderido ao substrato, geralmente em pedras e madeiras (Cummins et al., 2005; BAPTISTA et al., 2006). A presença desses organismos raspadores-escovadores nos demais substratos devese então à capacidade de utilização de MOPF não aderida firmemente, uma vez que eles não precisam necessariamente "raspar" o material aderido, e sim, "escovar" o material orgânico ligeiramente associado. Como MOPF é um recurso que pode estar disponível em todos os tipos de substrato, especialmente aqueles em áreas com menor velocidade na corrente de água, a distribuição dos organismos desta família não estaria restrita a substratos específicos dentro dos riachos.

A bacia do rio Pindaíba é composta por córregos alterados e preservados, alguns córregos são alterados devido à retirada da vegetação marginal e represamentos, enquanto que outros possuem a vegetação ribeirinha preservada por se localizarem em áreas de relevo acentuado, que impede o uso do solo por agricultura e pecuária. Dias-Silva et al. (2010) e SouZA et al. (2011), ao estudarem a bacia do rio Pindaíba tendo Heteroptera aquáticos e Baetidae como modelos biológicos, respectivamente, encontraram diferenças entre a riqueza, abundância e composição de espécies de córregos alterados e preservados. Isso comprova que a retirada da cobertura vegetal atua como um modificador do sistema. A retirada da vegetação pode ter acarretado no maior aporte de luz no sistema (Allan \& CASTILlo, 2007), permitindo maior diversidade de alimento, fato que pode ser responsável pela maior abundância de organismos nos trechos de segundas e terceiras ordens.

A maior riqueza de gêneros em trechos de terceira 
ordem corrobora os resultados de BAPTISTA et al. (2001a) e Corigliano et al. (2001), que encontraram a maior riqueza de Ephemeroptera em trechos intermediários dos córregos. De acordo com VANNOTE et al. (1980) e VISON \& Hawkins (1998), a alta diversidade de organismos em trechos intermediários de córregos e rios pode ser reflexo da elevada heterogeneidade e complexidade de hábitat desses locais.

Apesar de Ephemeroptera constituir apenas uma fração dos macroinvertebrados presentes nos ecossistemas aquáticos, a distribuição individual dos grupos funcionais alimentares de acordo com as ordens dos córregos e rios corroboram as predições do conceito do continuum fluvial.

Entretanto, ao contrário do que esperávamos, as ordens dos rios não foram bons preditores da composição funcional, uma vez que os grupos funcionais não foram estruturados pelas ordens dos córregos e rios. Já a composição taxonômica mostrou-se influenciada por esta classificação. A influência das ordens dos rios sobre as comunidades aquáticas foi abordada em outros trabalhos, como em Melo (2009), que relatou mudança abrupta na composição de macroinvertebrados entre as quarta e quinta ordens no rio do Carmo, em São Paulo. BAPTISTA et al. (2001b) encontraram quebra no padrão de organização da fauna de insetos aquáticos entre trechos de primeira à quarta ordem com relação aos trechos de quinta e sexta no rio Macaé, Rio de Janeiro. O tamanho dos córregos constitui-se, muitas vezes, do principal preditor da variação na estrutura taxonômica (HEINO et al., 2005), e muitas vezes não é linearmente relacionado com a sua ordem. Resultados encontrados em estudos da comunidade de Ephemeroptera e Trichoptera em córregos da transição Cerrado-Amazônia (SHImANo et al., 2010; NogueIRA et al., 2011, respectivamente) são exemplos deste padrão.

Porém a baixa influência da classificação hidrológica sobre a composição de Ephemeroptera pode ser reflexo de pequenas alterações ao longo dos cursos d'água. De acordo com o conceito de descontinuidade seriada (WARD \& STANFORD, 1983), as ações antrópicas como represas, poluição e erosão, agem como agentes de distúrbio, podendo causar descontinuidades dentro do continuum de um rio em pequenas escalas, o que pode levar à adição ou substituição de espécies, e/ou mudanças relativas de abundância (STANFORD \& WARD, 2001; AraúJo et al., 2009; KatAno et al., 2009).

De acordo com Buss et al. (2004), a distribuição de macroinvertebrados é influenciada mais pelo tipo de substrato do que pela integridade ambiental, qualidade da água ou período de amostragem. Porém, apesar dos substratos estudados diferirem com relação ao tipo, tamanho e disponibilidade de alimento, não encontramos relação entre a distribuição funcional dos Ephemeroptera e os tipos de substratos, o que refuta as nossas expectativas.

O fato da distribuição funcional nos substratos não ter refletido a mesma mudança observada na distribuição taxonômica, indica que os grupos funcionais alimentares podem não ser pré-estabelecidos baseados somente no tipo de substrato. Conforme Buss et al. (2004), muitos táxons podem colonizar mais que um substrato, mas, em geral, os substratos são utilizados por distintas comunidades de macroinvertebrados, provavelmente devido a adaptações similares para viver sobre diferentes condições de hábitat com relação à qualidade e quantidade do material orgânico. Além disso, as associações por substratos podem estar ligadas a outras condições que não exclusivamente as alimentares como, por exemplo, a demanda por oxigênio, abrigo contra predadores, adaptações à velocidade de água e estabilidade do substrato (FleCKER \& Allan, 1984). Organismos raspadores podem permanecer em folhiço em vez de pedra (e.g. Miroculis), ou um fragmentador pode ter associações com raiz em vez de folhiço (e.g. Fittkaulus).

Outros autores também verificaram variação de composição taxonômica de macroinvertebrados em função do tipo de substrato (KIKUCHI \& UIEDA, 2005). REZENDE (2007), por exemplo, ao trabalhar em córregos da Amazônia Central, concluiu que a composição de macroinvertebrados, assim como a abundância, é um parâmetro que pode estar associado ao fluxo da correnteza, uma vez que seriam necessários grupos com exigências ecológicas e comportamentais distintas nos ambientes de acordo com a velocidade da água.

As associações observadas na análise de espécies indicadoras demonstraram que poucos táxons apresentaram associação com o substrato baseado no grupo funcional. Por exemplo, os raspadores Thraulodes revelaram associação por pedras, enquanto que os fragmentadores Fittkaulus foram fiéis aos substratos vegetais.

Por outro lado, o também raspador Baetodes, além de se associar à pedra, esteve associado à folhiço em corredeira. Eles podem estar utilizando recursos de MOPF que são mais rapidamente incorporados a folhas em decomposição, como fungos e bactérias.

Além de agregar informações a respeito da classificação e distribuição funcional dos efemerópteros, os resultados desse estudo mostram que as comunidades estudadas são mais estruturadas quando se considera os táxons do que os grupos funcionais alimentares. Ao contrário do esperado, nossos resultados sugerem que não é possível a substituição da abordagem taxonômica pela funcional em se tratando de insetos efemerópteros. De acordo com CALlisto \& Esteves (1998), o estudo da estrutura trófica em guildas permite até orientar caminhos, mas são necessários estudos básicos de levantamento taxonômico para que seja possível avaliar efetivamente o papel das comunidades de macroinvertebrados bentônicos na ciclagem de nutrientes, fluxo de energia de ecossistemas aquáticos e demais trabalhos ecológicos. Sendo assim, acreditamos 
que ambas as abordagens são complementares e devem ser estudadas em conjunto.

Agradecimentos. Aos integrantes do Laboratório de Entomologia da Universidade do Estado de Mato Grosso, Campus de Nova Xavantina e às agências financiadoras FAPEMAT (processo $\mathrm{n}^{\circ}$ 098/2004) e PROCAD/CAPES (processo $n^{\circ}$ 109/2007).

\section{REFERÊNCIAS BIBLIOGRÁFICAS}

Allan, J. D. \& CASTILlo, M. M. 2007. Stream ecology: structure and function of running waters. Dordrecht, Springer. 436p.

AraúJo, F. G.; Pinto, B. C. T. \& Teixeira, T. P. 2009. Longitudinal patterns of fish assemblages in a large tropical river in southeastern Brazil: evaluating environmental influences and some concepts in river ecology. Hydrobiologia 618:89-107.

Assis, J. C. F.; Carvalho, A. L. \& Nessimian, J. L. 2004. Composição e preferência por microhábitat de imaturos de Odonata (Insecta) em um trecho de baixada do Rio Ubatiba, Maricá-RJ, Brasil. Revista Brasileira de Entomologia 48(2):273-282.

Baptista, D. F.; Buss, D. F.; Dorvillé, L. F. M. \& Nessimian, J. L. 2001a. Diversity and habitat preference of aquatic insects along the longitudinal gradient of the Macaé River Basin, Rio de Janeiro, Brazil. Revista Brasileira de Biologia 61(2):249-258.

Baptista, D. F.; Dorvillé, L. F.; Buss, D. F. \& Nessimian J. L. 2001b. Spatial and temporal organization of aquatic insects assemblages in the longitudinal gradient of a tropical river. Revista Brasileira de Biologia 61(2):295-304.

Baptista, D. F.; Buss, D. F; Nessimian, J. L.; Da-Silva, E. R.; Moraes Neto, A. H. A.; Carvalho, S. N.; Oliveira, M. A. \& Andrade, L. R. 2006. Functional feeding groups of Brazilian Ephemeroptera nymphs ultrastructure of mouthparts. Annales de Limnologie 42(2):87-96

Brasil. 1981. Projeto RADAMBRASIL, Folha SD 22. Goiás: geologia, geomorfologia, pedologia, vegetação, uso potencial da terra. Brasília, Ministério das Minas e Energia/Divisão de Publicação. 636p.

Buss, D. F.; Baptista, D. F.; Nessimian, J. L. \& Egler, M. 2004 Substrate specificity, environmental degradation and disturbance structuring macroinvertebrate assemblages in neotropical streams. Hydrobiologia 518:179-188.

Callisto, M. \& Esteves, F. A. 1998. Categorização funcional dos macroinvertebrados bentônicos em quatro ecossistemas lóticos sob influência das atividades de uma mineração de bauxita na Amazônia Central (Brasil). In: Neissimian, J. L. \& Carvalho, A. L. eds. Ecologia de Insetos Aquáticos. Rio de Janeiro, PPGEUFRJ. p.223-234.

ColWell, R. K. 2005. Estatistical estimation of species richness and shared species from samples (EstimateS), versão 7.5. Disponível em: $<$ http://viceroy.eeb.uconn.edu/estimates $>$. Acesso em: 29.04.2011.

Colwell, R. K.; Mao, C. X. \& Chang, J. 2004. Interpolating, extrapolating, and comparing in cidence-based species accumulation curves. Ecology 85(10):2717-2727.

Corigliano, M. C.; Gualdoni, C. M.; Oberto, A. M. \& Raffaini, G. B. 2001. Longitudinal distribution of the mayfly (Ephemeroptera) communities at the Chocancharava River Basin (Córdoba, Argentina). In: Dominguez, E. ed. Trends in Research in Ephemeroptera and Plecoptera. Tucuman, Kluwer Academic/ Plenum Publishers. p.89-95.

Cummins, K. W. 1973. Trophic relations of aquatic insects. Annual Review of Entomology 18:183-206.

1974. Structure and function of stream ecosystems. BioScience 24:631-641.

Cummins, K. W. \& Klug, M. J. 1979. Feeding ecology of stream invertebrates. Annual Review of Ecology and Systematics 10:147-172.

Cummins, K. W.; Merritt, R. W. \& Andrade, P. C. N. 2005. The use of invertebrate functional groups to characterize ecosystem attributes in selected streams and rivers in southeast Brazil. Studies on Neotropical Fauna and Environmental 40(1):71-90.

Cummins, K. W.; Merritt, R. W. \& Berg, M. B. 2008. Ecology and distribution of aquatic insects. In: MerRIt, R. W.; Cummins, K. W. \& BERG, M. B. eds. An introduction to the aquatic insects of North America. Duduque, Kendall/Hunt Publishing Company. p.105-122.
Dias-Silva, K.; Cabette, H. S. R.; Juen, L. \& De Marco Jr., P. 2010. The influence of habitat integrity and physical-chemical water variables on the structure of aquatic and semi-aquatic Heteroptera. Zoologia 27(6):918-930.

Domínguez, E.; Molineri, C.; Pescador, M. L.; Hubbard, M. \& Nieto, C. 2006. Ephemeroptera of South America. Moscow, Pensoft. $646 \mathrm{p}$

Dufrêne, M. \& LegendRE, P. 1997. Species assemblages and indicator species: the need for a flexible asymmetrical approach. Ecological Monographs 67(3):345-366.

Edmunds, JR. G. E.; Jensen, S. L. \& Berner, L. 1976. The Mayflies of North and Central America. Minnesota, University of Minnesota. 330p.

Flecker, A. S. \& Allan, J. D. 1984. The importance of predation, substrate and spatial refugia in determining lotic insect distribution. Oecologia 64(3):306-313.

Francischetti, C. N.; Da-Silva, E. R. \& Salles, F. F. 2001. A alimentação de ninfas de Caenis cuniana Froehlich, 1969 (Ephemeroptera, Caenidae) em um brejo temporário da restinga de Maricá, Estado do Rio de Janeiro. Boletim do Museu Nacional, Zoologia 446:1-6.

Francischetti, C. N.; Da-Silva, E. R.; Salles, F. F. \& Nessimian, J. L. 2004. A efemeropterofauna (Insecta: Ephemeroptera) do trecho ritral inferior do Rio Campo Belo, Itatiaia, RJ: composição e mesodistribuição. Lundiana 5(1):33-39.

Gotelli, N. J. \& Colwell, R. K. 2001. Quantifying biodiversity: procedures and pitfalls in the measurement and comparison of species richness. Ecology Letters 4(4):379-391.

Hannaford, M. J.; Barbour, M. T. \& Resh, V. H. 1997. Training reduces observer variability in visual-based assessments of stream habitat. Journal of the North American Benthological Society 16(4):853-860.

Heino, J.; Parviainen, J.; Paavola, R.; Jehle, M.; Louhi, P. \& Muotka T. 2005. Characterizing macroinvertebrate assemblage structure in relation to stream size and tributary position. Hydrobiologia 539:121-130

Hill, M. O. \& GaUCh, H. G. 1980. Detrended correspondence analysis, an improved ordination technique. Vegetatio 42(1-3):47-58.

Hilsenhoff, W. L. 2001. Aquatic orders of insects. In: Thorp, J. H. \& Covich, A. P. eds. Ecology and classification of North American freshwater invertebrates. San Diego, Academic Press. p.664680.

Katano, I.; Negishi, J. N.; Minagawa, T.; Doi, H.; Kanaguchi, Y. \& KAYABA, Y. 2009. Longitudinal macroinvertebrate organization over contrasting discontinuities: effects of a dam and a tributary. Journal of the North American Benthological Society 28(2):331-351.

Kikuchi, R. M. \& Uieda, V. S. 2005. Composição e distribuição dos macroinvertebrados em diferentes substratos de fundo de um riacho no município de Itatinga, São Paulo, Brasil. Entomología y Vectores 12(2):193-231.

McShaffrey, D. \& McCafferty, W. P. 1988. Feeding behavior of Rhithrogena pellucida (Ephemeroptera: Heptageniidae). Journa of the North American Benthological Society 7(2):87-99.

Melo, A. S. 2009. Explaining dissimilarities in macroinvertebrate assemblages among stream sites using environmental variables. Zoologia 26(1):79-84

Melo, A. S. \& Froenlich, C. G. 2001. Evaluation of methods for estimating macroinvertebrate species richness using individual stones in tropical streams. Freshwater Biology 46:711-721.

Merritt, R. W.; Cummins, K. W. \& Berg, M. B. 2008. An introduction to the aquatic insects of North America. Duduque, Kendall/ Hunt Publishing Company. 722p.

Minshall, G. W. 1984. Aquatic insect-substratum relationships. In: Resh, V. H. \& Rosenberg, D. M. C. P. eds. The Ecology of Aquatic Insects. Nova York, Praeger Publisher. p.358-399.

Mol, A. W. M. 1986. Harpagobaetis gulosus Gen. Nov., Spec. nov., a new mayfly from Suriname (Ephemeroptera:Baetidae). Zoologische Mededelingen 60(4):63-70.

Molineri, C. 2003. Revision of the South-American species of Leptohyphes Eaton (Ephemeroptera: Leptohyphidae) with a key to the nymphs. Studies on Neotropical Fauna and Environment 38(1):47-70.

Motta, R. L. \& Uieda, V. S. 2004. Diet and trophic groups of an aquatic insect community in a tropical stream. Brazilian Journal of Biology 64(4):809-817. 
Nogueira, D. S.; Cabette, H. S. R. \& Juen, L. 2011. Estrutura e composição da comunidade de Trichoptera (Insecta) de rios e áreas alagadas da bacia do rio Suiá-Miçu, Mato Grosso, Brasil. Iheringia, Série Zoologia 101(3):173-180.

Palmer, C.; O'Keeffe, J. \& Palmer, A. 1993. Macroinvertebrate functional feeding groups in the middle and lower reaches of the Buffalo River, eastern Cape, South Africa. II. Functional morphology and behaviour. Freshwater Biology 29(3):455-462.

Pardo, I. \& ARmitage, P. D. 1997. Species assemblages as descriptors of mesohabitats. Hydrobiologia 344:111-128.

Polegatto, C. M. \& Froehlich, C. P. 2003. Feeding strategies in Atalophlebiinae (Ephemeroptera: Leptophlebiidae), with considerations on scraping and filtering. In: GAINO, E. ed. Research Update on Ephemeroptera \& Plecoptera. Perugia, University of Perugia. p.55-61.

REZendE, C. F. 2007. Estrutura da comunidade de macroinvertebrados associados ao folhiço submerso de remanso e correnteza em igarapés da Amazônia Central. Biota Neotropica 7(2):300-305.

Rоот, R. B. 1967. The niche exploitation pattern of the blue-gray gnatcatcher. Ecological Monographs 37(4):317-350.

Salles, F. F. 2011. Lista das espécies de Ephemeroptera registradas para o Brasil. Disponível em: <http://ephemeroptera. br.googlepages.com/home>. Acesso em: 12.09.2011.

Salles, F. F.; Batista J. D. \& CABEtTe, H. S. R. 2004. Baetidae (Insecta: Ephemeroptera) de Nova Xavantina, Mato Grosso, Brasil: Novos registros e descrição de uma nova espécie de Cloeodes Traver. Biota Neotropica 4(2):1-8

SAVAGE, H. M. 1987. Biogeographic Classification of the Neotropical Leptophlebiidae (Ephemeroptera) based upon Geological Centers of Ancestral Origin and Ecology. Studies on Neotropical Fauna and Environment 22(4):199-222.
Schowalter, T. D. 2006. Insect ecology: An ecosystem approach. San Diego, Academic Press. 483p.

Shimano, Y.; Cabette, H. S. R.; Salles, F. F. \& Juen, L. 2010 Composição e distribuição da fauna de Ephemeroptera (Insecta) em área de transição Cerrado-Amazônia, Brasil. Iheringia, Série Zoologia 100(4):301-308.

Siegloch, A. E.; Froenlich, C. G. \& Kotzian, C. B. 2008. Composition and diversity of Ephemeroptera (Insecta) nymph communities in the middle section of the Jacuí River and some tributaries, southern Brazil. Iheringia, Série Zoologia 98(4):425-432.

Souza, H. M. L.; CABetTe, H. S. R. \& Juen, L. 2011. Baetidae (Insecta, Ephemeroptera) em córregos do cerrado matogrossense sob diferentes níveis de preservação ambiental. Iheringia, Série Zoologia 101(3):181-190.

Stanford, J. A. \& Ward J. V. 2001. Revisiting The Serial Discontinuity Concept. Regulated Rivers: Research \& Management 17:303-310.

Strahler, H. N. 1957. Quantitative analysis of watershed geomorphology. American Geophysical Union Transections 38:913-920.

Vannote, R. L.; Minshall, G. W.; Cummins, K. W.; Sedell J. R. \& Cushing, C. E. 1980. The River Continuum Concept. Canadian Journal of Fisheries and Aquatic Sciences 37:130-137.

Vison, M. R. \& Hawkins, C. P. 1998. Biodiversity of stream insects: Variation at local, basin and regional scales. Annual Review of Entomology 43:271-193.

Ward, J. V. \& Stanford, J. A. 1983. The serial discontinuity concept of lotic ecosystems. In: Fontaine, T. D. \& Bartell, S. M. eds. Dynamics of Lotic Ecosystems. Collingwood, Ann Arbor Scientific Publishers. p.29-42.

ZAR, J. H. 1999. Biostatistical analysis. Englewood Cliffs, PrenticeHall. 663p 